\title{
An Improved Quantum Stop-Wait Communication Protocol
}

\author{
Xincan Fan, Shaosheng Jiang and XiaojunWen* \\ School of Computer Engineering, Shenzhen Polytechnic, Shenzhen 518055, \\ People's Republic of China
}

\begin{abstract}
Based on the delocalized entanglement correlation and transience of transferring quantum information of quantum teleportation, an improved quantum stop-wait communication protocol for data link layer is presented. In the previous quantum stop-wait protocols, they only send the quantum acknowledgement frame (QACK) without sending quantum negative acknowledgement frame (QNACK). In this paper, we present an improved quantum stop-wait communication protocol that sends the negative frame QNACK as soon as possible when Bob detects the error of received data frame, instead of waiting for the retransmission time tout. As a result, the minimum time span between two successfully delivered data frames can be significantly reduced, and the communication time is shortened. It is shown that our protocol enhances the maximum throughput effectively and improves the communication efficiency in the data link layer.
\end{abstract}

Keywords: Quantum communication, stop-wait protocol, Data link layer

\section{Introduction}

In the early development phase of the computer network, communication network transmission quality is generally poor, and results in a higher error rate of data transmission. In this case, the data link layer protocol must address the issue of reliable transmission. Therefore, stop-wait protocol is the simplest but also the most basic communication protocol for data link layer. Nowadays, with the communication technology evolving rapidly, the error rate has declined significantly. However, the stop-wait protocol remains the foundation of some reliable transport protocols [1].

Recently, research on quantum communication technology has made great progress. In addition to quantum communication technology with high security, it has also many wonderful physical characteristics, which can provide special applications in communications [2-12], such as quantum entanglement collapse, EPR state, GHZ state, quantum teleportation and so on. Quantum teleportation has the following three characteristics: 1) the transfer of quantum information is instantly achieved. 2) It is not needed to know the location of each recipient when transferring quantum information. 3) The transfer process will not be blocked by any obstacles. By applying these features to the classical communication, the communication efficiency will be greatly improved. A typical approach is to send an acknowledgment frame via a quantum channel, in order to shorten the timeout retransmission time and increase the maximum throughput of the data link. Basing on this idea, in 2007, Zhou et al. proposed a quantum communication protocol for data link layer based on entanglement, which increases the maximum throughput effectively and improves the performance of stop-wait protocol for data link layer [13]. In 2013, they presented a three-party stop-wait quantum communication protocol based on GHZ states trying to solve the one-to-many multicast quantum communication protocol in data link layer [14].

*Corresponding author. E-mail addresses: szwxjun@sina.com 
In their presented protocols, when the recipient Bob receives the wrong data frame, he will not send any response and just wait for the sender Alice to re-send the correct data frame when timeout. This approach to deal with the error frames is simple, but it will affect the transmission efficiency. In this paper, we modified this approach by letting the receiver Bob immediately transmit a negative frame to the sender Alice when an error occurs, so that Alice may re-send the correct data frame as soon as possible.

\section{Communication Model}

In the previous stop-wait model, as figure 1.a shows, after Alice sending the DATA0 (frame number 0) with the starting a timeout timer, she will pause and wait for the confirmation from Bob. If the data frame is correctly received by Bob, it is delivered to the upper layer, and Bob will transmit an acknowledgment frame ACK1 to Alice. However, if some errors have occurred during the data0 transmission, Bob will discard this error data frame (DATA0) quietly. If the time exceeds tout (the re-transmission time set by timeout timer) and she still does not receive the acknowledgment frame from Bob, Alice may re-transmits previously sent data0.

In our improved stop-wait model, as figure 1.b shows, if some errors have occurred during the data0 transmission, instead of discarding this error data frame (DATA0) quietly, Bob will send a negative frame (NACK0) to Alice, in order to let Alice re-transmit the frame DATA0 as soon as possible.

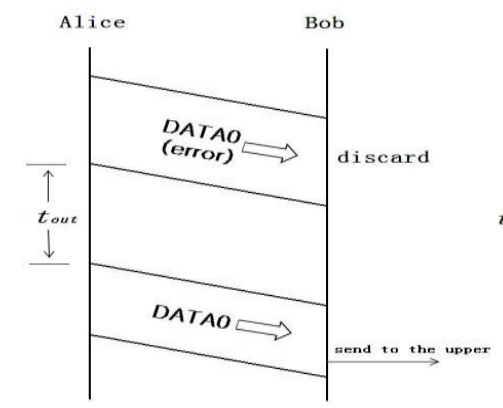

a. The Previous Protocol Model

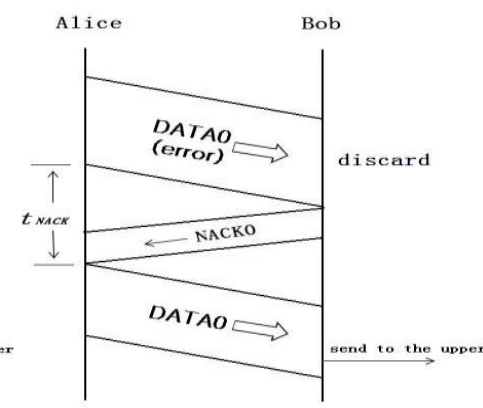

b. The Improved Protocol Model

Figure 1. The Stop- Wait Communication Protocol Model

\section{QACK Frame and QNACK Frame}

This paper intends to use quantum teleportation to send an acknowledgment frame or a negative frame. To distinguish between these two frames, the two recipients Alice and Bob agree the acknowledgment frame ACK (particle C) in the sate as

$$
|\phi\rangle_{A C K}=\frac{1}{\sqrt{3}}|0\rangle+\sqrt{\frac{2}{3}}|1\rangle
$$

As well as the negative frame NACK (particle C) in the state as

$$
|\phi\rangle_{N A C K}=\frac{1}{\sqrt{5}}|0\rangle+\frac{2}{\sqrt{5}}|1\rangle \cdot
$$

Notably, the particle $\mathrm{C}$ is prepared and is hold by the receiver Bob.

The actual data link is divided into busy time and leisure time. Supposed that Alice and Bob share EPR entangled state as

$$
|\phi\rangle_{A B}=\frac{1}{\sqrt{2}}\left(|01\rangle_{A B}-|10\rangle_{A B}\right)
$$

At the leisure time, and Bob transfer QACK frames or QNACK frames at the busy time, in order to improve the communication efficiency. 
On receiving the data frame from Alice, the receiver Bob checks whether the data frame has a transmission error or not. If wrong, Bob sends a QNACK frame to Alice through the quantum entanglement channel, otherwise sends a QACK frame. The quantum returning frames from Bob and Charlie involve the following two cases:

1). Sending QACK frames from Bob

The particle $\mathrm{C}$ and the EPR pair $\mathrm{AB}$ compose a quantum system as follows:

$$
\begin{gathered}
|\phi\rangle_{A B} \otimes|\phi\rangle_{C}=\frac{1}{2}\left[\left(-\frac{1}{\sqrt{3}}|0\rangle-\sqrt{\frac{2}{3}}|1\rangle\right)_{A}\left|\psi^{-}\right\rangle_{B C}+\left(-\frac{1}{\sqrt{3}}|0\rangle+\sqrt{\frac{2}{3}}|1\rangle\right)_{A}\left|\psi^{+}\right\rangle_{B C},\right. \\
\left.+\left(\sqrt{\frac{2}{3}}|0\rangle+\frac{1}{\sqrt{3}}|1\rangle\right)_{A}\left|\phi^{-}\right\rangle_{B C}+\left(-\sqrt{\frac{2}{3}}|0\rangle+\frac{1}{\sqrt{3}}|1\rangle\right)_{A}\left|\phi^{+}\right\rangle_{B C}\right]
\end{gathered}
$$

Where $\left|\psi^{ \pm}\right\rangle_{B C}=\frac{1}{\sqrt{2}}\left(|01\rangle_{B C} \pm|10\rangle_{B C}\right)$ and $\left|\phi^{ \pm}\right\rangle_{B C}=\frac{1}{\sqrt{2}}\left(|00\rangle_{B C} \pm|11\rangle_{B C}\right)$ are the four Bell states which can be distinguished by measuring.

In the case of sending QACK frames from Bob, Bob prepares the particle $\mathrm{C}$ in the state $_{|\phi\rangle_{A C K}}=\frac{1}{\sqrt{3}}|0\rangle+\sqrt{\frac{2}{3}}|1\rangle$ at first, then jointly measures particle B and particle $\mathrm{C}$ with Bell Base. The set time Tlater, Alice measures the particle A which will be in one of the four quantum states as follows: $\left(-\frac{1}{\sqrt{3}}|0\rangle-\sqrt{\frac{2}{3}}|1\rangle\right)_{A},\left(-\frac{1}{\sqrt{3}}|0\rangle+\sqrt{\frac{2}{3}}|1\rangle\right)_{A},\left(\sqrt{\frac{2}{3}}|0\rangle+\frac{1}{\sqrt{3}}|1\rangle\right)_{A}$, $\left(-\sqrt{\frac{2}{3}}|0\rangle+\frac{1}{\sqrt{3}}|1\rangle\right)_{A}$ with the probabilities $1 / 4$. It shows that Bob has received the data frame from Alice correctly if any one of these quantum states appears.

2). Sending QNACK frames from Bob

The particle $\mathrm{C}$ and the $\mathrm{EPR}$ pair $\mathrm{AB}$ compose a quantum system as follows:

$$
\begin{gathered}
|\phi\rangle_{A B} \otimes|\phi\rangle_{C}=\frac{1}{2}\left[\left(-\frac{1}{\sqrt{5}}|0\rangle-\frac{2}{\sqrt{5}}|1\rangle\right)_{A}\left|\psi^{-}\right\rangle_{B C}+\left(-\frac{1}{\sqrt{5}}|0\rangle+\frac{2}{\sqrt{5}}|1\rangle\right)_{A}\left|\psi^{+}\right\rangle_{B C},\right. \\
\left.+\left(\frac{2}{\sqrt{5}}|0\rangle+\frac{1}{\sqrt{5}}|1\rangle\right)_{A}\left|\phi^{-}\right\rangle_{B C}+\left(-\frac{2}{\sqrt{5}}|0\rangle+\frac{1}{\sqrt{5}}|1\rangle\right)_{A}\left|\phi^{+}\right\rangle_{B C}\right]
\end{gathered}
$$

where $\left|\psi^{ \pm}\right\rangle_{B C}=\frac{1}{\sqrt{2}}\left(|01\rangle_{B C} \pm|10\rangle_{B C}\right)$ and $\left|\phi^{ \pm}\right\rangle_{B C}=\frac{1}{\sqrt{2}}\left(|00\rangle_{B C} \pm|11\rangle_{B C}\right)$ are the four Bell states which can be distinguished by measuring.

In the case of sending QNACK frames from Bob, Bob prepares the particle C in the state $|\phi\rangle_{N A C K}=\frac{1}{\sqrt{5}}|0\rangle+\frac{2}{\sqrt{5}}|1\rangle$ at first, then jointly measures particle B and particle $\mathrm{C}$ with Bell Base. At the set time $\tau$, Alice measures the particle A which will be in one of the four quantum states as follows: $\left(-\frac{1}{\sqrt{5}}|0\rangle-\frac{2}{\sqrt{5}}|1\rangle\right)_{A},\left(-\frac{1}{\sqrt{5}}|0\rangle+\frac{2}{\sqrt{5}}|1\rangle\right)_{A},\left(\frac{2}{\sqrt{5}}|0\rangle+\frac{1}{\sqrt{5}}|1\rangle\right)_{A},\left(-\frac{2}{\sqrt{5}}|0\rangle+\frac{1}{\sqrt{5}}|1\rangle\right)_{A}$ with the probabilities of $1 / 4$. It shows that Bob have not received the data frame from Alice correctly if any one of these quantum states appears.

As stated above, the number of the Alice's measurement results about particle A is eight. The correlation between Alice's measurement results and the cases of QACK or QNACK frames from Bob is shown in Table 1. If the quantum states can be destroyed, it is feasible to differentiate the eight quantum states, which does not violate the quantum no-cloning theorem $[15,16]$. 
Table 1. The Correlation between Alice's Measurement Results and the Cases of QACK and QNACK Frames from Bob

\begin{tabular}{|c|c|}
\hline $\begin{array}{c}\text { The cases of QACK or QNACK } \\
\text { frame }\end{array}$ & Alice's measurement results \\
\hline $\begin{array}{c}\text { Case I: Sending QACK frames } \\
\text { from Bob }\end{array}$ & $\left(-\frac{1}{\sqrt{3}}|0\rangle-\sqrt{\frac{2}{3}}|1\rangle\right)_{A},\left(-\frac{1}{\sqrt{3}}|0\rangle+\sqrt{\frac{2}{3}}|1\rangle\right)_{A},\left(\sqrt{\frac{2}{3}}|0\rangle+\frac{1}{\sqrt{3}}|1\rangle\right)_{A}\left(-\sqrt{\frac{2}{3}}|0\rangle+\frac{1}{\sqrt{3}}|1\rangle\right)_{A}$ \\
\hline $\begin{array}{c}\text { Case II: Sending QNACK } \\
\text { frames from Bob }\end{array}$ & $\left(-\frac{1}{\sqrt{5}}|0\rangle-\frac{2}{\sqrt{5}} \mid 1\right)_{A},\left(-\frac{1}{\sqrt{5}}|0\rangle+\frac{2}{\sqrt{5}}|1\rangle\right)_{A}\left(\frac{2}{\sqrt{5}}|0\rangle+\frac{1}{\sqrt{5}}|1\rangle\right)_{A},\left(-\frac{2}{\sqrt{5}}|0\rangle+\frac{1}{\sqrt{5}}|1\rangle\right)_{A}$ \\
\hline
\end{tabular}

\section{Improved Quantum Stop-Wait Communication Protocol for Data Link Layer}

Within the set time $\tau$, Bob can receive data frame from Alice and accomplish the transmission of QACK frame or QNACK frame. Using the transmission and the detection method of acknowledgment frame above, the improved stop-wait quantum communication protocol involves the following steps:

\section{The sender Alice:}

(S1) Taking out a data frame from the host, and sending the data frame to the sending buffer.

(S2) V (S) $\leftarrow 0$, \{the initialization of the sending state variable\}.

(S3) $\mathrm{N}(\mathrm{S}) \leftarrow \mathrm{V}$ (S), \{ writing the value of the sending state variable into the sending sequence number $\}$.

(S4) Sending out the data frame from the sending buffer, \{the copy of the data frame is still kept in the sending buffer .

(S5) At measure time $\tau$, performing quantum measurement, according to Table 1, to determine whether the QACK frame or QNACK frame is sent back or not. If the case I, taking out the next new data frame from the host, V (S) $\leftarrow[1-\mathrm{V}$ (S)], \{updating the sending state variable into the next sequence number\}, going to (S3); If the case II, going to (S4), \{re-transmitting data frame .

The receiver Bob:

(R1) $\mathrm{V}(\mathrm{R}) \leftarrow 0, \quad$ the initialization of the receiving state variable $\}$

(R2) Waiting

(R3) On receiving a data frame, Bob verifies whether the data frame has a transmission error or not. If wrong, he performs relevant quantum measurement and sends QNACK frame, and goes to (R2); otherwise, he performs the next step.

(R4) If $\mathrm{N}(\mathrm{S})=\mathrm{V}(\mathrm{S})$, Bob performs the next step; otherwise, he discards the data frame, and goes to (R2).

(R5) Deliver the data portion of the received data frame to the host.

(R6) $\mathrm{V}(\mathrm{R}) \leftarrow[1-\mathrm{V}(\mathrm{R})]$, performing relevant quantum measurement and sending QACK frame.

\section{Performance Analysis}

As for the classical stop-wait protocol for data link layer, suppose that $t f$ is the transmission delay of a data frame, tp is the propagation delay from Alice (Bob) to Bob (Alice); tpr is the time Bob spend on dealing with data frame from Alice; ta is the transmission delay of Bob's QACK or QNACK frame; tpr is also the time Alice spend on dealing with QACK or QNACK frame from Bob. 


\section{(1) Compare To The Classical Stop-Wait Protocol For Data Link Layer}

In the classical stop-wait protocol, suppose that the minimum time span between two successfully delivered data frames from Alice to Bob is tT, then

$$
t_{T}=t_{f}+t_{\text {out }}=t_{f}+2 t_{p}+2 t_{p r}+t_{a}
$$

Suppose that the transmission error probability for each data is $p$, and the acknowledgment frames don't go wrong and the number of retransmissions isn't limited, so the average time of delivering correctly a data frame from Alice to Bob and Charlie is

$$
t_{a v}=t_{T}+(1-p) \sum_{i=1}^{\infty} i p^{i} t_{T}=\frac{t_{T}}{1-p}
$$

The maximum throughput for data link layer is

$$
\lambda_{\max }=\frac{1}{t_{a v}}=\frac{1-p}{t_{T}}
$$

In our improved stop-wait quantum communication protocol, the propagation delay tp of QACK or QNACK frame from Bob to Alice is not needed because the instantaneous characteristic of quantum teleportation. So the maximum throughput for data link layer in quantum protocol is

$$
\lambda_{Q \max }=\frac{1-p}{t_{T}-t_{p}}
$$

Comparing Eq. (8) with Eq. (9), we get

$$
\frac{\lambda_{Q \max }}{\lambda_{\max }}=\frac{t_{T}}{t_{T}-t_{p}}>1
$$

Eq. (10) shows that, as the previous quantum stop-wait protocol, the maximum throughput for data link layer in our protocol is obviously higher than that in the classical stop-wait protocol.

\section{(2) Compare To The Previous Quantum Stop-Wait Protocol For Data Link Layer}

In the previous quantum stop-wait protocols for data link layer, they only send the acknowledgement frame QACK without sending negative frame QNACK. If Alice does not receive QACK frame in the set time tout (re-transmission time), she will re-transmit the data frame. In the practical applications, tout is slightly larger than the required average time, that from beginning to send data frame to receive confirmation frame in the normal condition, this means

$$
t_{\text {out }}>t_{p}+2 t_{p r}+t_{a}
$$

So, in our quantum stop-wait protocol,

$$
t_{N A C K}=t_{p}+2 t_{p r}+t_{a}<t_{\text {out }}
$$

In our protocol, suppose the minimum time span between two successfully delivered data frame from Alice to Bob is tQT,

$$
t_{Q T}=t_{f}+t_{N A C K}<t_{T}
$$

So the maximum throughout for data link layer in our protocol is

$$
\lambda_{Q \max }^{\prime}=\frac{1-p}{t_{Q T}-t_{p}}
$$

Comparing Eq. (9) with Eq. (14), we get 


$$
\frac{\lambda_{Q \max }^{\prime}}{\lambda_{Q \max }}=\frac{t_{T}-t_{p}}{t_{Q T}-t_{p}}>1
$$

Eq. (15) shows that, compared to the previous quantum stop-wait protocols, the maximum throughput for data link layer in our protocol is higher too, because the re-transmission time from tout reduce to tNACK.

(3)About the measure time $\tau$

At the measure time $\tau$, Alice will measure her particle A, in order to judge whether Bob had sent the QACK or QNACK to her. The tis made up by the time tpr (Bob spends on dealing with data frame) and ta (the transmission delay of Bob's QACK or QNACK frame):

$$
\tau=t_{p r}+t_{a}
$$

\section{Conclusion}

By utilizing the delocalized entanglement correlation and transience of transferring quantum information of quantum teleportation, the previous quantum stop-wait protocols for data link layer improved the maximum throughput compared with classical stop-wait protocol. However, these quantum stop-wait protocols only send the quantum acknowledgement frame QACK without sending quantum negative acknowledgement frame QNACK. Therefore, we present an improved quantum stop-wait protocol by adding the negative acknowledgement frame QNACK when Bob detects the received data frame error. Based on this principle, when errors occurred in the received data frame, Alice will receive a quantum negative acknowledgement frame QNACK from Bob, rather than waiting for the set time tout (re-transmission time). In the practical applications, tNACK < tout, therefore both the transmission efficiency and the maximum throughput for data link layer are improved.

\section{Acknowledgements}

The work is supported by the Guangdong Provincial Natural Science Foundation (Grant No.S2013010015471), and the Shenzhen Basic Research Project (Grant No.JCYJ20120617140737337, Grant No.JCYJ20130331151803073).

\section{References}

[1] X. R. Xie, "Computer Network, 5th edn Pushing House of Electronic Industry", Beijing, (2008).

[2] M. Nielsen and I. Chuang, "Quantum Computation and Quantum Information", Cambridge University Press, Cambridge, (2000).

[3] Z. Zhao, Y. A. Chen, A. N. Zhang, T. Yang, H. J. Briegel, J. W. Pan, "Nature”, vol. 430, no. 54, pp. (2004).

[4] P. Garicia-Fernandez, E. Fernandez-Martinez, E. Perez and D. J. Santos, "Quantum Inf. Computer", vol. 5, no. 1 , (2005).

[5] G. Stix, "Sci. Am.", vol. 292, no. 78, (2005).

[6] T. Zhang, X. F. Mo, Z. F. Han and G. C. Guo, "Phys. Lett. A", vol. 372, no. 3957, (2008).

[7] B. Qi, W. Zhu, L. Qian and H. K. Lo, "New J. Phys", vol. 12, no. 103042, (2010).

[8] P. W. Shor and J. Preskill, "Phys. Rev. Lett.", vol. 85, no. 441, (2000).

[9] G. H. Zeng and C. H. Keitel, "Phys. Rev. A", vol. 65, no. 042312, (2002).

[10] X. J. Wen and Y. Liu, “Opt. Commun”, vol. 275, no. 278, (2007).

[11] X. J. Wen, X. M. Niu, L. P. Ji and Y. Tian, “Opt. Commun”, vol. 282, no. 666, (2009).

[12] X. J. Wen, Y. Tian, and X. M. Niu, "Physica Scripta", vol. 81, no. 055001, (2010).

[13] N. R. Zhou, G. H. Zeng, L. H. Gong and S. Q. Liu, “Acta Phys. Sin.”, vol. 56, no. 5066, (2007).

[14] N.R. Zhou, H. L. Cheng and H. L. Qing, "Int J Theor Phys", vol. 52, no. 811, (2013).

[15] N. R. Zhou, G. H. Zeng, F. C. Zhu and S.Q. Liu, "J. Shanghai Jiaotong Univ.”, vol. 40, no. 1885, (2006).

[16] A. Peres, "Phys. Lett.", vol. 128, no. 19, (1988). 